\title{
Incidentalomas, SPEW, and VOMIT—radiological dyspepsia?
}

\author{
Adrian Brady ${ }^{1,2}$
}

Received: 1 February 2020 / Revised: 5 March 2020 / Accepted: 30 March 2020 / Published online: 29 April 2020

(C) European Society of Radiology 2020

\section{Key Points}

- Incidental findings are an inevitable part of radiology reporting.

- Detection of incidental findings may be beneficial, but may result in unnecessary further investigation, and potential harm to patients.

- Incidental findings should be reported in context, with an attempt to offer guidance as to their significance, and the need to investigate further.

\section{Introduction}

\section{$\mathrm{CT}=$ computed tomography}

Incidentaloma = "an imaging abnormality in a healthy, asymptomatic patient, or an imaging abnormality in a symptomatic patient, where the abnormality was not apparently related to the patient's symptoms" [1]. First used to describe non-functioning incidental findings of adrenal nodules in 1982 [2]

GNC $=$ German National Cohort

VOMIT $=$ Victim of Modern Imaging Technology [3]

SPEW = Scans Propagating Exponential Workload [4]

Since the discovery of $\mathrm{x}$-rays 125 years ago, imaging technology and capabilities have developed hugely, often faster than the associated clinical knowledge. We frequently identify imaging findings, the significance or meaning of which is unknown. The implications of imaging findings for therapeutic options or patient outcomes may be unclear. Complex difficulties can surround radiological incidental findings, our responsibilities for reporting these, and referrer issues about what to do upon receipt of such a report. This mismatch between our ability to find abnormalities and our understanding of what to do about those findings may accelerate with increasing use of more-sensitive imaging techniques.

Adrian Brady

adrianbrady@me.com

1 Radiology Department, Mercy University Hospital, Cork T12 WE28, Ireland

2 Department of Radiology, School of Medicine, University College Cork, Cork, Ireland
As the use and capabilities of diagnostic imaging grow, it's appropriate to consider the interrelated issues of incidental findings, SPEW and VOMIT as features of our current environment of widespread imaging utilization, increasing patient empowerment, fear and danger of litigation, and ultimate responsibility to maximize benefit while minimizing harm.

\section{Benefits and negative consequences of incidental finding detection/reporting}

Most incidentally detected findings are unlikely to be relevant clinically, but sometimes their detection can be beneficial or even life-saving [5]. However, the investigations often triggered by detection of incidental findings, or by a radiologist's recommendations for further investigation, may subject a patient to additional hospital attendances and imaging, long-term follow-up, unnecessary or inappropriate treatment, including surgery, and potential actual physical harm (up to and including death). Significant anxiety for the patient may be incurred. Financial consequences can include wasteful healthcare expenditure, patient costs, and deleterious implications for future medical and life insurance $[1,6,7]$. Otherwise healthy individuals can be turned into anxious patients by the identification of findings never destined to harm them [8].

In 2002, William Casarella (a radiologist) reported his own experience of this phenomenon, writing: "the only 'normal' patient is one who has not yet undergone a complete workup." CT colonography as part of a routine annual check-up led to detection of a number of incidental findings. "Our observant radiologists saw them all." Further investigation of these led to a series of increasingly invasive investigations, for no ultimate significant health benefit [9]. Thus, he became a 
perfect example of a VOMIT (victim of modern imaging technology). The term was initially coined by a pediatric neurosurgeon (who also wrote, in the context of patients investigating their own symptoms, that "the internet is the most potent anxiety provoking system ever devised") [3].

SPEW (Scans Propagating Exponential Workload) is another acronym that describes the sequence which can develop when a radiologist reporting incidental findings suggests further investigations, which can then develop into a cascade similar to that experienced by Casarella, with much of the work done ultimately proving futile or even harmful [4].

Depending on the clinical situation, it can be the deliberate act of a wise radiologist to not report a clearly benign finding that might otherwise trigger unnecessary investigation on the part of a referrer, who might be less familiar with the abnormality. It has been suggested that refraining from reporting a benign simple renal cyst might be analogous to an internal medicine physician not mentioning an insignificant skin lesion in a report of a physical examination [10].

\section{How frequent (and significant) are "incidental findings"?}

"The more we image, the more we will find" [5]. As medical imaging grows inexorably, and the resolution of imaging modalities improves, the problem of irrelevant incidental findings will also grow [1].

Pulmonary nodules may be identified in up to $50 \%$ of chest CTs in smokers and up to $25 \%$ in non-smokers [11]. Incidental findings are identified in up to $70 \%$ of CT colonography studies (clinically significant incidental findings are identified in $5-16 \%$ in asymptomatic patients [10]), while renal and hepatic incidental findings are reported in about $15 \%$ of abdominal CTs. Thyroid US will find asymptomatic nodules in two thirds of patients [11].

The prevalence of malignancy is $<5 \%$ for incidental findings in the brain, parotid, and adrenal. Ten to $20 \%$ of prostatic and colonic incidental findings prove to be malignant, as do about $25 \%$ of renal, thyroid, and ovarian and $42 \%$ of breast incidental findings [1].

A recent report of patients with adrenal incidentalomas followed 969 such lesions (and a control group) for a mean of 8.9 years. All-cause mortality was significantly higher in those with adrenal incidentalomas (36.4\% vs. $31.6 \%)$. Unexpected adrenal lesions were associated with significantly higher incidences of malignancy, diabetes, heart failure, peripheral vascular disease, renal disease, and chronic pulmonary disease. The authors concluded that adrenal incidentalomas may represent a clinically valuable biomarker [12]. However, the lack of specificity of diseases associated with them makes it difficult to draw clear conclusions about the appropriate investigations which should result from detection of such abnormalities.

Some radiological findings may not truly be incidental, in that they represent underlying disease or abnormality, but may not require further investigation or therapeutic intervention at the time of reporting. An example of this is uncomplicated colonic diverticulosis in the asymptomatic adult population, which has been reported in $42 \%$ of subjects in a study of a Western population cohort undergoing MRI [13]. Reporting this finding may represent comprehensive assessment of imaging by the radiologist, but may lead to unnecessary patient anxiety or a false belief that further tests are required, if the patient has no relevant symptoms.

Secondary interpretations of imaging studies performed elsewhere are increasing in volume, especially in tertiary referral institutions, and particularly for oncological indications. In one institution, these have recently been reported to lead to recommendations for further imaging in $10 \%$ of cases. Many of these recommendations related to the primary reason for referral, but some arose from unrelated initial findings, including incidental findings. Changes in clinical management resulted from further imaging in $31.2 \%$ of cases [14].

\section{So why do we report incidental findings?}

1. Because we can, and feel we should.

2. To be sure (to reduce uncertainty) — many doctors and patients are unwilling to accept uncertainty, even when the chances of a serious diagnosis are extremely unlikely [10].

3. Risk aversion - "better to be safe than sorry" $[10,11]$.

4. Fear of litigation/defensive practice. Society seems to be more attentive to too little than to too much care and intervention $[11,15]$.

5. Lack of clear-cut algorithms for diagnosis and treatment strategies [10].

6. Lack of confidence (to ignore findings we consider of no consequence).

7. To follow published guidelines, where they exist [16].

8. For financial gain; in some healthcare funding models, it may be in the financial interest of the radiologist to recommend further imaging. Additionally, hospitals and medical equipment vendors/owners may find their interests served by more investigation [11].

\section{Why not follow every incidental finding?}

"What is the responsible use of information nobody asked for" $[10]$ ? 
The rationale behind pursuing incidental findings (if one is conceived or given) is usually to exclude serious (including malignant) disease. Most incidental findings will not cause harm to the patient; however, further investigation to establish this frequently results in "a cascade of testing that is costly, provokes anxiety, exposes patients to radiation unnecessarily, and may even cause morbidity" [10].

In an ideal world, economic cost would be irrelevant in this context. Medical certainty, in so far as it is achievable, would be pursued for all findings. But we do not live in an ideal world; healthcare costs are rising well in excess of inflation, and imaging services and their costs are rising faster than other medical technologies [17]. It's incumbent on those who use (often limited) resources to deploy them for the best possible effect. Reliance on evidence-based medicine to govern investigation is central to this [10]. The fact that a condition could exist does not mean we should pursue every unlikely possibility; not every 30 year-old with chest pain needs a CT pulmonary angiogram. "Biological plausibility.....is a necessary, but not sufficient basis for decision-making" [8]. Probability, not possibility, must support demands for further investigation.

Equally, clinical context must be taken into account; in patients with significant co-morbidities or limited life expectancy, pursuit of reported abnormalities may be of no relevance [10]. In a young, healthy person, further investigation of the same abnormality might be warranted.

Not all reports identifying unexpected incidentalomas are unhelpful. Failure to comply with recommendations for further investigation can delay diagnosis and treatment, lead to unhelpful non-imaging testing, and expose both referrers and radiologists to legal liability if outcomes are bad [18]. Many renal carcinomas are now diagnosed as incidental findings on imaging performed for other reasons [5]. Furthermore, increasingly cross-sectional imaging is being used as a non-specific "catchall" investigation in patients without clear clinical diagnoses. If no specific possible diagnosis is being considered prior to imaging, which findings are truly incidental? [5].

Undoubtedly, much reporting of incidental findings constitutes overdiagnosis, identification of an abnormality that will never cause symptoms or death of the patient [11]. Shaughnessy has written that "Overselling of medical care as the solution to many aspects of the human condition, leads to over diagnosis" [8]. Overdiagnosis has been described as a public health problem [11]. However, overdiagnosis and under-diagnosis are simply two areas on a line with no clearly defined perfect center point; striving to avoid one will inevitably lead to an element of the other [5].

\section{How often do we suggest further imaging?}

It must be emphasized that most recommendations for further imaging are entirely appropriate and helpful to patients, representing application of radiological knowledge to guide referrers to valuable information they need, ultimately benefiting patients and limiting inappropriate imaging usage. However, this is not always the case. Not every radiologist reports the same imaging study in the same way. Rates of recommendations for further investigation are highly variable among institutions, and among radiologists within the same institution $[11,15]$. Furthermore, radiologists are poor at predicting their own rate of making such recommendations, but are somewhat better at predicting the rates of colleagues [17]. Despite this variation, it seems such recommendations are becoming more common. From 1995 to 2008, the frequency of recommendations for additional imaging in one large US academic hospital more than doubled, without an increase in positive results [19].

Cochon and colleagues considered the frequency with which follow-up recommendations are made by radiologists. Reported rates range from 8 to $37 \%$. Overall, they identified such recommendations in $12.2 \%$ of over 300,000 studies. Modality-wise, CT had the highest likelihood of follow-up suggestions (other than in neuroradiology, where MRI was highest). Follow-up recommendations were most common in older patients and in females, and lower in inpatients [15]. Other studies have shown that for all types of breast imaging, additional radiological investigations are recommended in 15\% of cases. Radiologist experience did not correlate with frequen$\mathrm{cy}$, and inter-radiologist probabilities within subspecialties varied from 2.8-fold to 6.7-fold [15]. Conversely, other studies have reported a direct (negative) correlation between radiologist experience and frequency of follow-up recommendations, with a decrease of about $15 \%$ per decade of experience $[11,19]$, emphasizing the likelihood that some recommendations for further imaging are based on lack of confidence.

Furthermore, if additional (or follow-up) investigations are requested by the referrer, it's unlikely that the same radiologist will always report subsequent images $[1,15]$. Thus, the emphasis of different reports will vary; what one radiologist considers worth highlighting might be thought irrelevant by another [15]. Additionally, radiologists (and referrers) may vary in their knowledge of and adherence to evidence-based guidelines, influencing comparability of reports [15]. Lack of standardization of follow-up recommendations may compromise clinical decision-making, and worsen the adverse consequences [15]. Close relationships between referrers and radiologists are more common in highly specialized practices, and are helpful, as both parties learn one another's use of language, style of practice, and habits. However, most radiology reporting is not done in such a tight, close one-to-one environment [15]. Regardless of such relationships, referrers may feel obliged to act upon recommendations for additional imaging made by radiologists, in order to protect themselves from legal liability challenges [17]. 
We should not overestimate the size of the problem. Lee found that only $8 \%$ of high-cost (CT, MR, US, or nuclear medicine) examinations were prompted by a radiologist's recommendation [20]. Nor should this relatively low figure promote complacency; this percentage gives no indication of how often radiologists made recommendations for further investigations which were not acted upon.

\section{What should we do about incidental findings?}

Some guidelines exist for further investigation and management of incidental findings, but these are often directed at radiologists rather than referrers [1]. The American College of Radiology (ACR) has published position papers on incidental findings on thoracic CT [16], in the adrenal, liver, pancreas, and kidneys. Referring clinicians may not be aware of these, or may choose not to abide by them. Furthermore, many such guidelines derive from academic medical centers, where the pressure to achieve diagnostic certainty may be more intense (for reasons of teaching, the greater severity of illness that may be seen in academic centers, more-ready availability of advanced investigative technologies, and the needs of research and greater sub-specialization, which can skew assessment of the likely significance of incidental findings) [10]. Guidelines from such sources may not be helpful in practice settings that may be very different.

An additional source of reported incidental findings that can cause particular difficulty is imaging utilized as part of screening studies, often in asymptomatic individuals. There are no internationally agreed recommendations regarding management of incidental abnormalities found in these circumstances; this is a particular issue with the growth of lung cancer screening using chest CT. We do not always know which findings are of no clinical consequence and which are significant enough to require further evaluation [21]. In a recent study of CT lung cancer screening, only $1.8 \%$ of the pulmonary findings led to additional evaluation, while $15.3 \%$ of cardiovascular findings resulted in referral for further testing [22]. The German National Cohort (GNC) is a large population-based cohort study investigating the development of common chronic diseases in volunteers, a subgroup of whom undergo whole-body magnetic resonance imaging, which inevitably leads to detection of some incidental findings. A strategy for managing such findings divides them into those requiring notification (urgent or otherwise) to the patient, and those not requiring notification. Notified patients are then encouraged to seek medical advice and further workup, if required. Study participants have reported high stress levels after receiving notification of incidental findings, prior to knowing their ultimate significance [6]. Similar negative effects on emotional well-being, finances, and work or activities were reported by patients receiving notifications of incidental findings requiring further investigation in a UK Biobank study [7].

It has been suggested that incidental findings on imaging studies should be considered a complication or side effect of the investigation, and that patients' consent should be obtained before imaging investigations, the possibility of incidental findings having been explained and discussed. In a model of healthcare that shares the making of diagnostic and management decisions between patient and doctor, this makes sense [1], but the relationship remains unbalanced. Doctors are generally better positioned to evaluate the likelihood of incidental findings being significant than many patients, who may inevitably rely on their advice.

Demand for medical "certainty" that drives much SPEW is a demotion of the role of the physician in patient care, from the position of a knowledgeable provider of information and guidance, to a conduit for channeling endless investigation until certainty is achieved. This approach risks eliminating doctors from the chain - if we simply make more and more investigations available until 100\% diagnoses are reached, why can we not be replaced by AI, algorithms, and decision pathways?

\section{What about patients reading reports?}

Patients increasingly read their own clinical radiology reports, either via online patient portals or after receiving copies directly. This is an empowering development and enhances patients' ability to participate directly in decision-making about their own care. In the context of incidental findings, it also has potential negative consequences. How are patients to understand the significance of reported findings that may be of no clinical significance, if they are also not provided with context and explanation? Some patient advocates have called for "patient summaries" to be added to radiology reports, or for two versions of a report to be issued, one for the referrer, and one for the patient (using language understandable to lay people) [23]. This would undoubtedly help avoid unnecessary fear about what may be insignificant findings on radiology studies, but in a world where demand for radiology services substantially exceeds our ability to meet that demand, the time required to provide these additional patient-focused elements of reports is unlikely to be available. Nonetheless, given that we know patients now frequently read their radiology reports directly, radiologists should be aware of how findings that to us may be inconsequential (e.g., sigmoid diverticulosis, Bosniak 1 renal cysts) may be terrifying to lay readers. We can allay much of that fear by considering including explanatory comments ("of no clinical consequence," "typical for the patient's age," "a normal anatomical variant") in our reports when reporting incidental findings we know require no further investigation or action. This may lengthen our reports slightly, 
and may be seen as redundant by some referrers, but may ultimately save everybody unnecessary worry and time.

\section{What would be helpful?}

There are no all-encompassing actions that will solve the conundrum of what findings we should report or ignore, and how far we should go in pursuing the meaning of apparently incidental radiologic findings. Thresholds for reporting incidental findings will inevitably vary among radiologists, and the extent to which their reporting will be welcomed by patients and their referrers will be as individual as the patients themselves. Nonetheless, there are a few aspirations that, if they were available or adopted, might guide radiologists and referrers, and reduce negative consequences and anxiety for patients:

1. Devising methods to make recommendation rates among radiologists with varying thresholds and experience more uniform.

2. Standardization (where possible) of definitions of incidental finding thresholds, led by radiological professional societies [1].

3. Feedback reports showing rates of follow-up recommendations for individual radiologists [15].

4. Inclusion of a feedback method (email, telephone, etc.) in radiology reports, by which referrers could contact the reporting radiologist directly to seek clarification or advice about findings.

5. Education to promote use of decision-support tools and adherence to evidence-based imaging guidelines among referrers and radiologists $[15,18,24]$. The probability of a condition must be emphasized in medical education, and encouraged in practice [8].

\section{A few take-home messages}

1. For referrers: don't shoot the messenger. The radiologist didn't make the findings, he/she just reported them.

2. For radiologists:

A. Radiologists ARE doctors. It's incumbent on us to contextualize findings where possible, to suggest next steps, and to identify those findings which do not matter. A moment adding an explanation to a report may save a lot of time and anxiety in the long run.

B. When reporting findings that may not be pertinent to the current clinical question, STOP and THINK about their importance and the possible consequences of reporting them before proceeding.
3. For referrers AND radiologists: context is everything. An adrenal nodule in a demented 95 -year-old is not the same as one in a healthy 25 -year-old.

4. Remember: while it is useful to always have perfection as a goal, given the human condition, perfection in medicine is unattainable.

Funding information The authors state that this work has not received any funding.

\section{Compliance with ethical standards}

Guarantor The scientific guarantor of this publication is Adrian Brady.

Conflict of interest The author of this manuscript declares no relationships with any companies whose products or services may be related to the subject matter of the article.

Statistics and biometry No complex statistical methods were necessary for this paper.

Informed consent Written informed consent was not required for this study because no individual patients or imaging studies were relevant to the paper.

Ethical approval Institutional Review Board approval was not required because no individual patients or imaging studies were relevant to the paper.

\section{Methodology}

- Observational

\section{References}

1. O'Sullivan JW, Muntinga T, Grigg S, Ioannidis JPA (2018) Prevalence and outcomes of incidental imaging findings: umbrella review. BMJ 361:k2387. https://doi.org/10.1136/bmj.k2387

2. Geelhoed GW, Druy EM (1982) Management of the adrenal "incidentaloma". Surgery 95:866-874

3. Hayward R (2003) VOMIT (victims of modern imaging technology) - an acronym for our times. BMJ 326:1273. https://doi.org/10. 1136/bmj.326.7401.1273

4. Houston M (2019) Incidental findings lead to risk of unnecessary harm. Irish Times. https://www.irishtimes.com/newspaper/archive/ 2019/0730/Pg030.html\#Ar03000:1AF2AB1D32B9. Accessed 20 Jan 2020

5. Maskell G (2018) Think before you scan. BMJ 362:k3754. https:// doi.org/10.1136/bmj.k3754

6. Hegedüs P, von Stackelberg O, Neumann C et al (2019) How to report incidental findings from population whole-body MRI: view of participants of the German National Cohort. Eur Radiol 29: 5873-5878. https://doi.org/10.1007/s00330-019-06077-z

7. Gibson LM, Littlejohns TJ, Adamska L et al (2018) Impact of detecting potentially serious incidental findings during multimodality imaging. Wellcome Open Res 2:114. https:/www.ncbi. nlm.nih.gov/pmc/articles/PMC6024231/. Accessed 5 March 2020

8. Shaughnessy AF, Slawson DC (2019) Getting at the roots rather than pruning the branches of overdiagnosis. BMJ Opinion. https:// blogs.bmj.com/bmj/2019/03/15/getting-at-the-roots-rather-thanpruning-the-branches-of-overdiagnosis/. Accessed 5 April 2020 
9. Casarella WJ (2002) A patient's viewpoint on a current controversy. Radiology 224:927

10. Berland LL, Silverman SG, Gore RM et al (2010) Managing incidental findings on abdominal CT: white paper of the ACR Incidental Findings Committee. J Am Coll Radiol 7:754-773

11. Chojniak R (2015) Incidentalomas: managing risks. Radiol Bras 48(4):1X-X. https://doi.org/10.1590/0100-3984.2015.48.4e3

12. Taya M, Paroder V, Bellin E, Hacamatı LB (2019) The relationship between adrenal incidentalomas and mortality risk. Eur Radiol 29: 6245-6255. https://doi.org/10.1007/s00330-019-06202-y

13. Storz C, Rothenbacher T, Rospleszcz S et al (2019) Characteristics and associated risk factors of diverticular disease assessed by magnetic resonance imagingin subjects from a Western general population. Eur Radiol 29:1094-1103. https://doi.org/10.1007/s00330018-5687-5

14. Heinz SA, Kwee TC, Yakar (2020) Recommendations for additional imaging of abdominal imaging examinations: frequency, benefit and cost. Eur Radiol 30:1137-1144. https://doi.org/10.1007/ s00330-019-06382-7

15. Cochon LR, Kapoor N, Carrodeguas E et al (2019) Variation in follow-up imaging recommendations in radiology reports: patient, modality and radiologist predictors. Radiology 291:700-707. https://doi.org/10.1148/radiol.2019182826

16. Munden RF, Carter BW, Chiles C et al (2018) Managing incidental findings on thoracic $\mathrm{CT}$ : mediastinal and cardiovascular findings. A White Paper of the ACR Incidental Findings Committee. J Am Coll Radiol 15(8):1087-1096

17. Kapoor N, Lacson R, Cochon LR, Boland GW, Khorasani R (2019) Radiologists' self-assessment versus peer assessment of perceived probability of recommending additional imaging. J Am Coll Radiol. https://doi.org/10.1016/j.jacr.2019.11.022

18. Hendee WR, Backer GJ, Borgstede JP et al (2010) Addressing overutilization in medical imaging. Radiology 257:240-245

19. Sistrom CL, Dreyer KJ, Dang PP et al (2009) Recommendations for additional imaging in radiology reports: multifactorial analysis of 5.9 million examinations. Radiology 253:453-461

20. Lee SI, Saokar A, Dreyer KJ, Weilburg JB, Thrall JH, Hahn PF (2007) Does radiologist recommendation for follow-up with the same imaging modality contribute substantially to high-cost imaging volume. Radiology 242:857-864

21. Kauczor H-U, Baird A-M, Blum TG et al (2020) ESR/ERS statement paper on lung cancer screening. Eur Radiol. https://doi.org/10. 1007/s00330-020-06727-7

22. Morgan L, Choi H, Reid M, Khawaja A, Mazzone PJ (2017) Frequency of incidental findings and subsequent evaluation in low-dose computed tomographic scans for lung cancer screening. Ann Am Thorac Soc 14(9):1450-1456

23. Briers E (2019) The patient perspective - sharing expectations and experiences - Part 2. Presentation at ECR 2019, ESR Patient Advisory Group (ESR-PAG) session on Communicating the role of the radiologist - best practices to manages patient expectations, Vienna, March 2, 2019

24. ESR iGuide. European Society of Radiology. http:// nationaldecisionsupport.com/esriguide/index.html. Accessed 20 Jan 2020

Publisher's note Springer Nature remains neutral with regard to jurisdictional claims in published maps and institutional affiliations. 